Biometrical journal - Accepted paper

\title{
Bayesian analysis of censored response data in family-based genetic association studies
}

Fabiola Del Greco M. ${ }^{1}$, Cristian Pattaro ${ }^{1}$, Cosetta Minelli ${ }^{2}$, John R. Thompson ${ }^{3}$

\begin{abstract}
Biomarkers are subject to censoring whenever some measurements are not quantifiable given a laboratory detection limit. Methods for handling censoring have received less attention in genetic epidemiology, and censored data are still often replaced with a fixed value. We compared different strategies for handling a left-censored continuous biomarker in a family-based study, where the biomarker is tested for association with a genetic variant, $\mathbf{S}$, adjusting for a covariate, $\mathbf{X}$. Allowing different correlations between $\mathbf{X}$ and $\mathbf{S}$, we compared: simple substitution of censored observations with the detection limit followed by a linear mixed effect model (LMM); Bayesian model with non-informative priors; Tobit model with robust standard errors; the mutiple imputation (MI) with and without $\mathbf{S}$ in the imputation followed by a LMM. Our comparison was based on real and simulated data in which $20 \%$ and $40 \%$ censoring were artificially induced. The complete data was also analysed with a LMM. In the MICROS study, the Bayesian model gave results closer to those obtained with the complete data. In the simulations, simple substitution was always the most biased method; the Tobit approach gave the least biased estimates at all censoring levels and correlation values; the Bayesian model and both MI approaches gave slightly biased estimates but smaller root mean square errors. On the basis of these results the Bayesian approach is highly recommended for candidate gene studies; however, the computationally simpler Tobit and the MI without $\mathbf{S}$ are both good options for genome-wide studies.
\end{abstract}

\section{Keywords}

Bayesian methods; Genetic association studies; Left-censored data; Multiple imputation; Tobit model

\footnotetext{
${ }^{1}$ Center for Biomedicine, EURAC research, Bolzano, Italy, E-mail: fabiola.delgreco@eurac.edu

${ }^{2}$ Respiratory Epidemiology, Occupational Medicine and Public Health, NHLI, Imperial College, London, UK

${ }^{3}$ Department of Health Sciences, University of Leicester, Leicester, UK
} 


\section{Introduction}

The problem of analysing censored data arises naturally in many fields of medical research. Much of the methodological work on this topic has focused so far on survival analysis, but similar issues are encountered in studies where the laboratory assay does not enable the measurement of biomarker levels over the full range of its possible values. Such values that cannot be quantified are often denoted as non-detectable, ND, and they have also been recognised as censored data, see e.g. [Taylor, 1987] who denoted the LOD as the instrumental limit of detection, sometimes called censoring threshold. This type of biological data are often subject to left-censoring (i.e. ND values falling below LOD), due to the difficulty of quantifying very low concentrations of the biomarker. Replacing those censored data with a single fixed value induces a distributional mass concentrated between zero and the minimal observable value. This is illustrated in Figure 1, which shows the distribution of a nearly Gaussian variable when $20 \%$ or $40 \%$ censored values are replaced by the value zero.
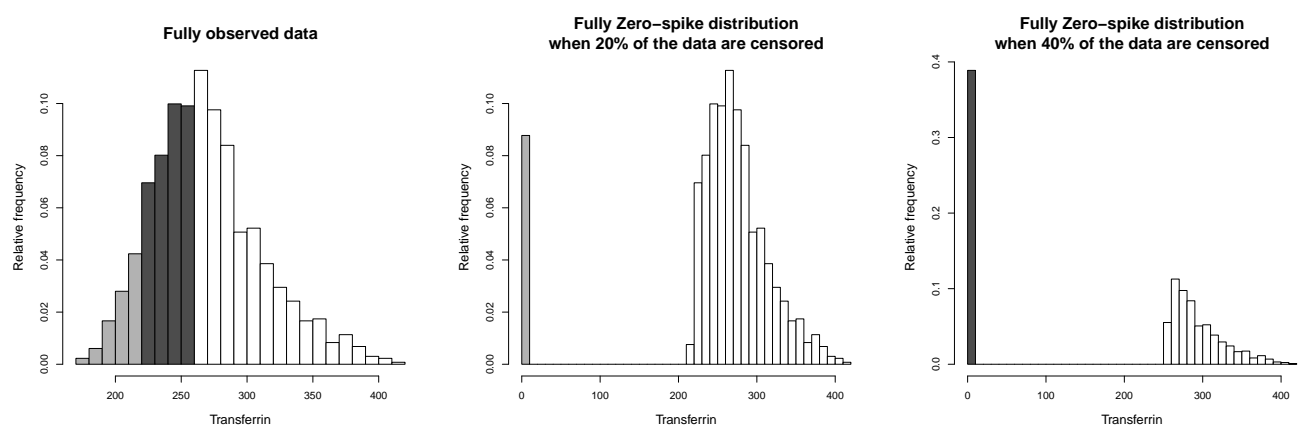

Figure 1: Left-censoring may induce spike-at-zero data. Left panel: distribution of serum transferrin levels in the 131 family trios of the MICROS study [Pattaro et al., 2007] when data are fully observed; light and dark gray indicate the proportion of values that would be imputed to the zero-spike in case of $20 \%$ and $40 \%$ censoring rate, respectively. Middle panel: spike-at-zero distribution corresponding to $20 \%$ censoring rate. Right panel: spike-at-zero distribution corresponding to $40 \%$ censoring rate.

Genetic association studies can be classified into two groups: candidate gene studies, where a single or few genetic variants are tested for association with a biomarker, and genome-wide association studies (GWAS) where millions of genetic variants are scanned for association with the biomarker of interest. While the purpose of candidate gene studies is to confirm a biological hypothesis, the aim of GWAS is to uncover novel genes, that were not known before to be associated with a given biomarker. Although the issue of dealing with censored data in these contexts has been extensively investigated in the last decade [Epstein et al., 2003, Diao et al., 2004, Diao and Lin, 2006, Fang, 2006, Li et al., 2010], the omission of ND values from the analyses is still common although it has been demonstrated that this may lead to biased estimates [Little and Rubin, 2002, Schafer, 1997], particularly 
when the proportion of ND values is above 10\% [Lubin et al., 2004].

Methods for handling censored data in family-based genetic association studies fall into three broad categories: (i) simple substitution, (ii) distribution, and (iii) robust methods. In this paper we do not consider alternative methods for handling censored data through categorization of the outcome, for example using ordinal regression. These approaches tend to cause a loss of power and require a different interpretation of the fitted coefficients.

In simple substitution, ND values are replaced by a fixed value (e.g. zero; LOD; LOD/2; $\mathrm{LOD} / \sqrt{2})$.

In distribution methods, a model is assumed for the full data and it is fitted using maximum likelihood or probability plot procedures [Casella and Berger, 2002] that are modified to allow for the censoring. An alternative fitting procedure, known as imputation, replaces the ND values by randomly sampled or expected values and then fits the model as if the data were complete. When this replacement is repeated at each iteration of the fitting model procedure, the process can be considered as a data augmentation approach [Tanner, 1991]. In a single imputation only one replacement is undertaken; it guarantees unbiased, or minimally biased, estimates for censoring rates lower than 30\%, but leads to biased variance estimates, probably due to the fact that the sampling variability is not considered [Lubin et al., 2004]. In a multiple imputation (MI) procedure several plausible sets of replacements for the missing values are created. Each imputed dataset is analysed separately and the results are averaged [Schafer, 1999]. In the last few years, specific MI procedures have been included in many statistical programs as the default option because MI has been demonstrated to decrease bias and increase efficiency over single imputation [White and Carlin, 2010]. On the other hand, it is well known that the MI procedure is not always the best approach [Schafer, 1999], and in some cases it provides valid estimates only if the censoring rate is under 50\% [Lubin et al., 2004]. Although MI has been used for dealing with censored data in survival analysis (Hsu, 2007), there is no evidence on performance of the MI in genetic association studies with dependent observations due to family structure. An important issue in MI is the choice of the variables to include in the imputation model. It has been shown that all variables that will be used in the final analysis should be included in the imputation model [Rubin, 1996, Schafer et al., 2002]. In genetic association studies this implies that the imputation model should include any epidemiological variable that may be associated with the outcome (e.g. age or sex) as well as the genetic variant itself. Furthermore, confounding factors specific to genetic associations, such as population stratification [Price et al., 2006], also need to be modelled in order to provide unconfounded estimates of the effect of the genetic variant. Within the Bayesian framework, Gibbs sampling offers a convenient way of obtaining MI replacements as they can be taken from different parts of the Monte Carlo Chain when fitting the imputation model. A Bayesian MI procedure was proposed for longitudinal and clustered data with response and predictor missing values by Schafer and Yucel [Schafer and Yucel, 2002, Yucel, 2008].

A third approach is the so-called robust analysis which includes parametric methods (e.g. the Tobit model [Tobin, 1958]) with robust standard errors, semiparametric models (e.g. the censored quantile regression [Portonoy, 2003]), and semiparametric variance-component models [Diao and Lin, 2006]. The Tobit variance-component method assumes that the cen- 
sored data follow a multivariate normal distribution [Epstein et al., 2003]. Since the violation of this assumption can lead to type I error inflation, power reduction, and bias of the parameter estimate, the Tobit variance-component model was modified into the copula variance-component method, that directly models the non-normal distribution using Gaussian copulas [Li et al., 2006]. The semiparametric variance-component model is an extension of the previous model, which allows the modelling of a censored trait within families without any distributional assumption. Despite the flexibility of the variance-component framework and the fact that censoring is a common problem in genetic association studies, these particular variance-component models are rarely used. Similarly, the censored quantile regression, which has been extended for use with dependent data [El Ghouch and Van Keilegom, 2009], is not widely used.

The aim of this paper is to evaluate a Bayesian approach for the analysis of censored data with a family structure in the context of a genetic association study, by comparing it against alternative solutions. The paper is organised as follows. In Section 2, we introduce data structure with notation, and describe different methods for censoring data analysis. For comparison, all methods are contrasted against the situation when the whole data range is available. In Section 3, the proposed methods are compared in real data from the MICROS study [Pattaro et al., 2007], a cross-sectional study where a completely observed biomarker is artificially left-censored. In Section 4, all methods are compared based on simulated scenarios of left-censored family data. Discussion and concluding remarks are presented in Section 5 .

\section{Methods for censored data analysis}

This section is organized as follows: in subsection 2.1, we define the theoretical framework and describe the linear mixed effect model (LMM) typically used in family-based association studies; in subsection 2.2 we describe the simple substitution approach; in subsection 2.3 the Tobit model with robust standard errors estimation is described; subsection 2.4 presents a full Bayesian modelling solution; and subsection 2.5 introduces the MI procedure where the Bayesian modelling is combined with LMM association analysis. All approaches for censored data analysis described in this section are summarized in Table 1.

\subsection{Family-based association studies and the linear mixed effect model (LMM)}

Let's start by considering a biomarker $\mathbf{y}$ fully observed on $N$ subjects from $n$ families of fixed size $k$, so that $n \times k=N$. The biomarker can be defined as a vector $\mathbf{y}=$ $\left(\mathbf{y}_{1}^{\prime}, \mathbf{y}_{2}^{\prime}, \ldots, \mathbf{y}_{i}^{\prime}, \ldots, \mathbf{y}_{n}^{\prime}\right)^{\prime}$, where each $\mathbf{y}_{i}=\left(y_{i 1}, y_{i 2}, \ldots, y_{i j}, \ldots, y_{i k}\right)$ is the vector of individual observations $j$ within family $i$. We model $\mathbf{y}_{i}$ by a $k$-dimensional multivariate normal distribution $\operatorname{MVN}_{k}\left(\boldsymbol{\mu}_{i}, \mathbf{V}_{i}\right)$, where $\boldsymbol{\mu}_{i}=\mathbf{X}_{i} \boldsymbol{\beta}$. The design matrix, $\mathbf{X}_{i}$, will include the intercept, the covariates and measured genetic variants. In its general form, the model assumes the variance-covariance matrix to be the sum of an additive genetic component $(A)$, a common environment component $(\mathrm{C})$ and an independent error component (E) (ACE structure); this can be expressed as $\mathbf{V}_{i}=\sigma_{g}^{2} \mathbf{K}_{i}+\sigma_{e}^{2} \mathbf{E}_{i}+\sigma_{r}^{2} \mathbf{I}$ [Visscher et al., 2004]. In this formulation, $\mathbf{K}_{i}$ 
is the matrix of the so-called 'relatedness coefficients' describing the pairwise genetic similarities and $\sigma_{g}^{2}$ is the additive genetic variance of all unmeasured genes that influence the biomarker level. $\mathbf{E}_{i}$ is a $k \times k$ matrix of ones and $\sigma_{e}^{2}$ is the component of variance associated with shared family environment. $\mathbf{I}$ is the $k \times k$ identity matrix and $\sigma_{r}^{2}$ is the residual variance.

Model fitting is often performed by means of the LMM

$$
\mathbf{y}=\mathbf{X} \boldsymbol{\beta}+\mathbf{b}+\boldsymbol{\epsilon}
$$

where $\mathbf{X}$ is the design matrix which includes the intercept and $p$ predictors, either environmental or genetic; $\boldsymbol{\beta}=\left(\beta_{0}, \beta_{1}, \ldots, \beta_{p}\right)^{\prime}$ is the vector of $p+1$ fixed effects coefficients; $\mathbf{b}=\left(\mathbf{b}_{1}^{\prime}, \mathbf{b}_{2}^{\prime}, \ldots, \mathbf{b}_{n}^{\prime}\right)^{\prime}$ is composed of $n k$-dimensional vectors of random effects, where $\mathbf{b}_{i}^{\prime} \sim N\left(\mathbf{0}, \sigma_{g}^{2} \mathbf{K}_{i}+\sigma_{e}^{2} \mathbf{E}_{i}\right)$; and $\boldsymbol{\epsilon}$ is the random error vector distributed as $N\left(\mathbf{0}, \sigma_{r}^{2} \mathbf{I}\right)$ [Schifano et al., 2012].

In the following, we will use the LMM over the fully observed data as term of comparison for all model solutions for censored data. In both application and simulations, the LMM was fitted using the $\mathrm{R}$ packages coxme and nlme (see Supporting Information).

Finally, we assume that some of the biomarker measurements fall below a known measurement threshold and are thus censored, such that the vector of observations $\mathbf{y}$ can be partitioned into observed, $\mathbf{y}_{o}$, and censored, $\mathbf{y}_{c}$, values.

\subsection{Simple substitution approach}

Simple substitution refers to the practice of replacing all censored observations $\mathbf{y}_{c}$ by a fixed value that could range from 0 to the LOD, and then regressing the biomarker against the covariates and the genetic variant by fitting a LMM to take into account the family structure, as described above.

\subsection{Tobit model with robust standard errors estimation}

The basic Tobit model [Tobin, 1958] was developed for independent censored observations. In our framework, this would correspond to the situation of $k=1$, that is the biomarker is measured on only one individual per family $(N=n)$, so that we can use $y_{i}$ in place of $y_{i j}$. The model considers a latent variable $y_{i}^{*}$ that describes the theoretical unobserved values for $y_{i} \leq \mathrm{LOD}$ and is equal to the observed ones whenever $y_{i}>\mathrm{LOD}$. Such latent variable is modelled as being dependent on the covariates and measured genetic variants, $y_{i}^{*}=\boldsymbol{\beta}^{\prime} \mathbf{x}_{i}+\epsilon_{i}$ where the $\epsilon_{i}$ is distributed as a $N\left(0, \sigma_{r}^{2}\right)$.

More generally, a convenient way to take into account the dependence among observations due to the family structure, is fitting a Tobit model with robust standard errors estimation. This method allows to deal with heteroscedasticity caused by the presence of correlated data [Wooldridge, 2002]. In the following application and simulations, the Tobit was fitted using the AER package in $R$, which has a robust option that provides sandwich estimates for the covariance matrix of parameters and, therefore, of the corresponding standard errors (see Supporting Information). 


\subsection{Full Bayesian approach}

Censoring represents a particular case of not at random missingness (Little and Rubin, 2002), commonly referred to as coarsening, i.e. the exact value of the variable is not observed, but it is only known to belong to the sample space where the true data are contained. The coarsening mechanism can be ignored in the full likelihood function when it is known in advance, as it occurs when the censoring threshold is fixed (Heitjan and Rubin, 1991; Heitjan, 1993). However, the observed-data likelihood function becomes very complicated in the presence of censoring, and its maximization is not straightforward even under the assumption of normality. For this reason, we introduce a Bayesian approach to estimate the unknown parameters and to generate imputed values.

An MCMC algorithm for fitting the model uses a form of data augmentation and can be divided into two steps. First, we estimate the censored values conditional on the current estimates of the model parameters and the observed values of the biomarker, then we update the parameter estimates by fitting the regression model to the full set of observed and estimated values of the biomarker. Data augmentation involves sampling from a series of conditional normal distributions and, provided that conjugate priors are chosen for the parameters, the posterior of $\boldsymbol{\beta}$ will be a multivariate normal distribution and only the variance components that account for family structure need updating by a numerical method such as Metropolis-Hastings or slice sampling [Gelfand et al., 1990, Schafer and Yucel, 2002, Gelman et al., 2004, Box and Tiao, 2011].

Following the framework described above, it is convenient to factorize the covariance matrix as $\mathbf{V}_{i}=\sigma_{r}^{2}\left(\lambda_{1} \mathbf{K}_{i}+\lambda_{2} \mathbf{E}_{i}+\mathbf{I}\right)$ where $\lambda_{1}=\left(\sigma_{g}^{2} / \sigma_{r}^{2}\right)$ and $\lambda_{2}=\left(\sigma_{e}^{2} / \sigma_{r}^{2}\right)$. Then, $\boldsymbol{\theta}=\left(\boldsymbol{\beta}, \sigma_{r}^{2}, \lambda_{1}, \lambda_{2}\right)$ represent the full set of parameters. Update $t$ in the MCMC algorithm can be represented as,

$$
\begin{aligned}
\mathbf{y}_{c i}^{(t)} & \sim \mathrm{P}\left(\mathbf{y}_{c i} \mid \mathbf{y}_{o i}, \boldsymbol{\theta}^{(t-1)}\right) \\
\boldsymbol{\theta}^{(t)} & \sim \mathrm{P}\left(\boldsymbol{\theta} \mid \mathbf{y}_{o i}, \mathbf{y}_{c i}^{(t)}\right) .
\end{aligned}
$$

Following Shafer and Yucel [Schafer and Yucel, 2002], proper prior distributions are defined for the parameters in $\boldsymbol{\theta}$. We use a multivariate normal prior $\operatorname{MVN}(\mathbf{M}, \mathbf{T})$ for $\boldsymbol{\beta}$, an inverse gamma prior $I G\left(a_{r}, b_{r}\right)$ for $\sigma_{r}^{2}$ and gamma priors $G\left(a_{1}, b_{1}\right)$ and $G\left(a_{2}, b_{2}\right)$ for $\lambda_{1}$ and $\lambda_{2}$ respectively, and we perform the augmentation of the censored values one at a time. Suppose that the $j^{\text {th }}$ member of family $i$ has a censored biomarker, $y_{i j}$, and let $\mathbf{y}_{i J}$ represent the observed and currently estimated biomarker values of all other members of that family. Partition the mean vector for the family into the mean for subject $j, \mu_{i j}$ and the mean for the other members of the family, $\boldsymbol{\mu}_{i J}$, and partition the variance matrix similarly as, $\mathbf{V}_{i}=\left(\begin{array}{cc}\mathbf{V}_{j j} & \mathbf{V}_{j J} \\ \mathbf{V}_{J j} & \mathbf{V}_{J J}\end{array}\right)$ [Flury, 1997]. The conditional distribution of $y_{i j}$ given $\mathbf{y}_{i J}$ is normal with $\mathrm{E}\left[y_{i j} \mid \mathbf{y}_{i J}, \boldsymbol{\theta}\right]=\mu_{i j}+\mathbf{V}_{j J} \mathbf{V}_{J J}^{-1}\left(\mathbf{y}_{i J}-\boldsymbol{\mu}_{i J}\right)$ and $\operatorname{Cov}\left[y_{i j} \mid \mathbf{y}_{i J}, \boldsymbol{\theta}\right]=\mathbf{V}_{j j}-\mathbf{V}_{j J} \mathbf{V}_{J J}^{-1} \mathbf{V}_{J j}$. Thus, each censored observation is replaced by a new value drawn from a univariate normal distribution truncated at the LOD. 
Assuming that all the regression coefficients are jointly normally distributed [Dipak et al., 2000], $\boldsymbol{\beta}$ can be drawn from

$$
\operatorname{MVN}\left(\left(\sum_{i=1}^{n} \mathbf{X}_{i}^{\prime} \mathbf{V}_{i}^{-1} \mathbf{X}_{i}+\mathbf{T}^{-1}\right)^{-1}\left(\sum_{i=1}^{n} \mathbf{X}_{i}^{\prime} \mathbf{V}_{i}^{-1} \mathbf{y}_{i}+\mathbf{M} \mathbf{T}^{-1}\right),\left(\sum_{i=1}^{n} \mathbf{X}_{i}^{\prime} \mathbf{V}_{i}^{-1} \mathbf{X}_{i}+\mathbf{T}^{-1}\right)^{-1}\right),
$$

given the augmented data. When the priors on the individual regression coefficients are independent univariate normal distributions, the components of $\boldsymbol{\beta}$ could be updated sequentially using draws from the corresponding univariate normal marginal posterior distributions.

Setting $\Omega_{i}=\left(\lambda_{1} \mathbf{K}_{i}+\lambda_{2} \mathbf{E}_{i}+\mathbf{I}\right)^{-1}$, the parameter $\sigma_{r}^{-2}$ turns to have a conditional distribution of a gamma random variable

$$
\sigma_{r}^{-2} \mid \boldsymbol{\beta}, \lambda_{1}, \lambda_{2}, \mathbf{y}_{i} \sim G\left(\frac{n k}{2}+a_{r}, \frac{1}{2} \sum_{i=1}^{n}\left(\mathbf{y}_{i}-\mu_{i}\right)^{\prime} \Omega_{i}\left(\mathbf{y}_{i}-\mu_{i}\right)+b_{r}\right),
$$

with the prior becoming vaguer as $a_{r}$ and $b_{r}$ tend to zero. Since the conditional distributions of $\lambda_{1}$ and $\lambda_{2}$ are not of a standard form a method of updating is required, such as slice sampling [Neal, 2003] (see Supporting Information).

Notice that, to simplify the algorithm, we omitted the estimation of a random coefficient related to the family structure. While this accelerates the MCMC, it is not expected to have major implications given its null expected value and given that the related covariance partition is already embedded in the model. The full MCMC algorithm is reported in the Supporting Information.

\subsection{Multiple imputation}

When different models are to be fitted to the same biomaker, for example when many genetic variants are to be tested for their effect on the same biomarker, then it may be more efficient to run a single preliminary Bayesian imputation model and to extract different sets of imputed biomarkers from widely separated parts of the MCMC output. Each of the sets of augmented values is then analysed using a LMM, as if there were no censoring. The combined estimate across the imputations is calculated by the simple average of the estimates from each data sets and its variance by the sum of the within-imputation and $(1+1 / M)$ the between-imputation variances. A small value of $M(2 \leq M \leq 10)$ is usually sufficient [Schafer, 1999] (see Supporting Information).

The imputation model should ideally incorporate all variables included in the final analysis [Schafer, 1999, Bell and Fairclough, 2013], which would mean to include the genetic variant under test. This would make the use of the MI approach computationally intensive in the context of GWAS that evaluates millions of genetic variants one at a time. However, such an approach may be feasible in the context of a candidate gene association study, where only a few genetic variants are considered.

\section{Genetic associations with serum transferrin levels in the MICROS study}

To empirically compare the methods described above, we analysed the association between serum transferrin levels and three genetic variants in the Microisolates in South Tyrol (MICROS) study, which is a population-based study carried out in 2002/03 on 1,325 
volunteers recruited from three alpine villages. Briefly, the study was a cross-sectional investigation aimed at investigating the genetic basis of human health and disease through the screening of several biomarkers and self-reported disease status [Pattaro et al., 2007]. Several biomarkers proved to be highly heritable [Marroni et al., 2008] and relevant genetic findings have been identified [Del Greco-M et al., 2011]. An important feature of the study was its extended pedigree connecting all participants in a single genealogical tree spanning several generations [Gögele et al., 2011]. Participants were $18-88$ year old, $56 \%$ were females and all of them gave written informed consent to study participation.

Transferrin is the carrier protein which binds most of the iron levels present in the serum. Hence, its concentration is an important proxy of the iron binding capacity involved in several essential metabolic pathways, and crucial for human health. On this sample, we previously performed a GWA analysis of serum transferrin levels [Pichler et al., 2011]. The genetic heritability of serum transferrin in the MICROS study, as estimated from a variance component model based on 304,383 genotyped variants using the GenABEL package in R, was equal to 0.35 . For the present work, we re-analysed three genetic variants that were genome-wide significant in the discovery analysis (see Table S2 of [Pichler et al., 2011]): rs3811647 in the transferrin $(T F)$ gene, rs12637730 in the RAB6B member RAS oncogene family $(R A B 6 B)$ gene, and rs6781238 in the carnitine deficiency-associated gene expressed in ventricle $3(C D V 3)$. To mimic a typical GWAS, where millions of variants are processed one at a time, we analysed the three selected variants separately.

Given that the structure of the MICROS pedigree is very irregular, with families composed of different number of subjects and with many missing relatives, we selected a subsample of 131 family trios, consisting of three siblings each. This design implies that the relatedness coefficients matrix $\mathbf{K}_{i}$ is equal to $\left(\begin{array}{ccc}1 & 0.5 & 0.5 \\ 0.5 & 1 & 0.5 \\ 0.5 & 0.5 & 1\end{array}\right)$ for each family $i$. This simplified structure enables to better control the formulation of the models and their comparison.

The 393 individuals included in the subsample had a mean age of 46 years (standard deviation, SD $=15$ ), and $53 \%$ were females. Serum transferrin was assessed over its full range, and approximately normally distributed with mean of $271 \mathrm{mg} / \mathrm{dl}$ and standard deviation of $43 \mathrm{mg} / \mathrm{dl}$ (Figure 1, left panel). This biomarker was strongly associated with age ( $\mathrm{p}$-value $<2 \times 10^{-16}$ ), with a correlation coefficient of -0.24 . Being age independent of any genetic variant, the analysed scenario is similar to that represented in Figure 2a, where the biomarker levels are determined by two independent variables: one genetic and one non-genetic. The three genetic variants had different minor allele frequencies (MAF, range: 0.19 to 0.35 ) and showed some pairwise correlation (linkage disequilibrium $r^{2}$ range: 0 to 0.35 ). Hence, the association between transferrin and one genetic variant at a time, was assessed in the fully observed transferrin values by using the LMM, where age was included as a covariate. The results of this model, reported in the first line of Table 2, confirmed the strong association between the three variants and the biomarker, despite the smaller sample size.

After introducing left-censoring of $20 \%$ and $40 \%$ of the observations (see middle and 
right panels in Figure 1), we applied in turn all five approaches for censored data analysis summarised in Table 1. The use of a fully observed dataset with artificially induced censoring allows us to investigate empirically how the different strategies perform in situations with different degrees of censoring, when the answer for the full data is known.

Table 1: Approaches followed to deal with a left-censored response variable in this work. Models fit the association between a left-censored biomarker $\mathbf{Y}$, a genetic variant $\mathbf{S}$ and a non-genetic covariate $\mathbf{X}$. In both real data application and simulations, all five models have been compared to a linear mixed model fitted to the fully observed data (our gold-standard).

\begin{tabular}{|l|l|l|}
\hline \multicolumn{1}{|c|}{ Method } & Abbreviation & \multicolumn{1}{c|}{ Description } \\
\hline 1. Simple substitution & Substitution & $\begin{array}{l}\text { Linear mixed effect model of } \mathbf{Y} \text { on } \mathbf{S} \text { and } \mathbf{X} \text { after replacement } \\
\text { of censored values with the limit of detection }\end{array}$ \\
\hline 2. Tobit model & Tobit & Tobit model, with robust standard errors, of $\mathbf{Y}$ on $\mathbf{S}$ and $\mathbf{X}$ \\
\hline 3. Bayesian analysis & Bayesian & Full Bayesian model with $\mathbf{S}$ and $\mathbf{X}$ \\
\hline 4. Multiple imputation with $\mathbf{S}$ & MI+S & $\begin{array}{l}5 \text { imputations with } \mathbf{S} \text { and } \mathbf{X} \text { in the Bayesian model followed by } \\
\text { linear mixed effect model of each new } \mathbf{Y} \text { on } \mathbf{S} \text { and } \mathbf{X}\end{array}$ \\
\hline 5. Multiple imputation without $\mathbf{S}$ & MInoS & $\begin{array}{l}5 \text { imputations with } \mathbf{X} \text { in the Bayesian model followed by } \\
\text { linear mixed effect model of each new } \mathbf{Y} \text { on } \mathbf{S} \text { and } \mathbf{X}\end{array}$ \\
\hline
\end{tabular}

In the Bayesian analysis, we set the genetic variance $\sigma_{g}^{2}=0.35$, corresponding to the observed genetic heritability and the residual variance $\sigma_{r}^{2}=0.65$; the initial values of the algorithm were based on estimating a linear regression model for the fully observed data; hyperparameters values were chosen in such a way as to induce vague priors; we fixed the starting variance ratio $\lambda_{1}=0.3$, as this corresponds to the genetic heritability (ratio between the biomarker variance explained by additive genetic effects and its total variance) of serum transferrin in the MICROS dataset. The Gibbs sampling algorithm was run for 5,500 iterations, with the first 500 discarded as burn-in. Convergence was checked graphically, and the posterior means were used as the point estimates. In the MI analyses, either with and without the genetic variant, we used 5 imputations taken from evenly spread points in the Monte Carlo chain, and each new dataset was regressed on the genetic variant and age using the LMM.

Results are reported in Table 2. The simple substitution approach led to estimates always smaller than those obtained with the complete data; whereas the Tobit model with robust standard errors gave the largest estimates, especially when the censoring rate was equal to 40\%. The Bayesian model and both MI approaches gave good estimates, which were similar each other and quite close to those from the complete data. For all methods and scenarios, we observed an increase of the bias with the percentage of censored value. The extent of the bias was not related to the MAF of the studied genetic variants. 
Table 2: Association between left-censored serum transferrin levels and three genetic variants, using age as a covariate.

\begin{tabular}{|c|c|c|c|c|c|c|c|}
\hline \multirow[b]{3}{*}{ Method } & \multirow[b]{3}{*}{ Censoring \% } & \multicolumn{6}{|c|}{ Genetic variant (gene, reference allele, allele frequency) } \\
\hline & & \multicolumn{2}{|c|}{$\begin{array}{c}\text { rs12637730 } \\
(R A B 6 B, \mathrm{~A}, 0.16)\end{array}$} & \multicolumn{2}{|c|}{$\begin{array}{c}\mathbf{r s 3 8 1 1 6 4 7} \\
(T F, \mathrm{~T}, 0.31)\end{array}$} & \multicolumn{2}{|c|}{$\begin{array}{c}\text { rs6781238 } \\
(C D V 3, \mathrm{~T}, 0.59)\end{array}$} \\
\hline & & $b(s e)$ & $p$-value & $b(s e)$ & $p$-value & $b(s e)$ & $p$-value \\
\hline Complete & 0 & $9.4(4.4)$ & $1.6 \times 10^{-2}$ & $16.9(3.4)$ & $3.3 \times 10^{-7}$ & $8.3(3.2)$ & $4.7 \times 10^{-3}$ \\
\hline \multirow{2}{*}{ 1. Substitution } & 20 & $7.8(4.0)$ & $2.6 \times 10^{-2}$ & $14.8(3.1)$ & $9.0 \times 10^{-7}$ & $8.2(2.9)$ & $2.3 \times 10^{-3}$ \\
\hline & 40 & $6.6(3.6)$ & $3.3 \times 10^{-2}$ & $12.1(2.8)$ & $7.8 \times 10^{-6}$ & $7.8(2.6)$ & $1.3 \times 10^{-3}$ \\
\hline \multirow[t]{2}{*}{ 2. Tobit } & 20 & $10.8(5.0)$ & $1.5 \times 10^{-2}$ & $18.2(3.7)$ & $4.4 \times 10^{-7}$ & $6.5(3.6)$ & $3.5 \times 10^{-3}$ \\
\hline & 40 & $11.0(5.6)$ & $2.5 \times 10^{-2}$ & $20.7(4.3)$ & $7.4 \times 10^{-7}$ & $11.3(4.0)$ & $2.4 \times 10^{-3}$ \\
\hline \multirow[t]{2}{*}{ 3. Bayesian* } & 20 & $9.7(4.0)$ & & $18.2(3.8)$ & & $7.7(3.9)$ & \\
\hline & 40 & $8.7(4.1)$ & & $21.6(4.0)$ & & $9.6(4.0)$ & \\
\hline \multirow[t]{2}{*}{ 4. $\mathrm{MI}+\mathrm{S}$} & 20 & $9.6(4.7)$ & $21 \times 10^{-2}$ & $18.8(3.9)$ & $8.4 \times 10^{-7}$ & $7.9(3.5)$ & $1.2 \times 10^{-2}$ \\
\hline & 40 & $8.6(5.1)$ & $4.6 \times 10^{-2}$ & $20.6(4.3)$ & $3.3 \times 10^{-7}$ & $8.6(3.8)$ & $1.2 \times 10^{-2}$ \\
\hline \multirow[t]{2}{*}{ 5. MInoS } & 20 & $9.8(4.8)$ & $2.1 \times 10^{-2}$ & $18.9(4.0)$ & $1.2 \times 10^{-6}$ & $7.6(3.5)$ & $1.5 \times 10^{-2}$ \\
\hline & 40 & $8.9(4.6)$ & $2.7 \times 10^{-2}$ & $20.4(4.4)$ & $1.8 \times 10^{-6}$ & $8.6(4.0)$ & $1.6 \times 10^{-2}$ \\
\hline
\end{tabular}

Abbreviations: Genetic effect (b); Standard error (se).

*For the Bayesian analysis, the posterior mean with its standard deviation are reported.

\section{Simulation}

We randomly generated 1,000 datasets of 100 families comprised of three siblings each, to reflect the same situation as in the empirical example above. In such families with three siblings it is not possible to differentiate between additive polygenic effects and common environmental effects, because all of the genetic correlations are equal as are all the correlations due to shared environment. Hence, we chose the AE representation in which the variancecovariance matrix within families is given by the sum of an additive polygenic component (A) and an independent error (E) terms, that is $\mathbf{V}_{i}=\sigma_{g}^{2} \mathbf{K}_{i}+\sigma_{r}^{2} \mathbf{I}$, where the relatedness coefficients matrix is the same as that defined in the real data example; moreover, we assumed the same genetic variance $\sigma_{g}^{2}=0.35$, and residual variance $\sigma_{r}^{2}=0.65$.

A genetic variant $\mathbf{S}$ with allele frequency $f=0.4$, such that it explained $2 \%$ of the variance in the biomarker, was generated from a binomial distribution $B(2,0.4)$. Whereas, a covariate $\mathbf{X}$ was generated from a normal distribution with a within-family covariance matrix equal to the identity matrix.

Two possible scenarios were analysed. In our first scenario (Figure 2a), we investigated three levels of correlation between $\mathbf{X}$ and $\mathbf{Y}, \rho_{X Y}=(0.2,0.5,0.8)$, and set $\rho_{X S}=0$. For the second scenario (Figure $2 \mathrm{~b}$ ), we fixed $\rho_{X Y}=0.2$ and $\rho_{X S}=(0.3,0.5)$. For each dataset, we set the LOD so as to produce $20 \%$ and then $40 \%$ left-censoring.

We performed the same analyses as in the real data example (see Table 1). In the Bayesian analysis, initial values of the regression coefficients were based on estimates from a linear regression analysis performed on the part of data that could be observed, that is, of the data above the LOD; we used the same relatedness matrix $\mathbf{K}_{i}$ and fixed the same starting variance ratio $\lambda_{1}$. Hyperparameters values were chosen in such a way as to induce vague priors. As the initial values were well-chosen we were able to use a burn-in of 500 iterations. The Gibbs sampling algorithm with slice sampling for $\lambda_{1}$ mixed very well and 
a)

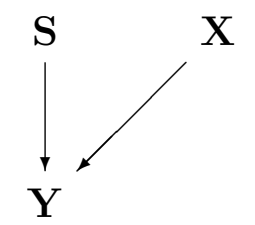

b)

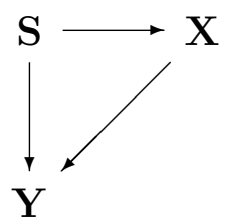

Figure 2: Study framework with a censored biomarker $\mathbf{Y}$, a covariate $\mathbf{X}$ and a genetic variant S. In scenarios: a) $\mathbf{S}$ and $\mathbf{X}$ are both associated with $\mathbf{Y}$, but $\rho_{X S}=0$; b) $\mathbf{S}$ and $\mathbf{X}$ are both associated with $\mathbf{Y}$ and they are positively correlated with each other, $\rho_{X S}>0$.

a run of 5,500 iterations converged with a sampling error for the coefficient of the genetic variant below 0.01. Convergence was checked graphically for representative scenarios before running the repeated simulations. Posterior means were used as point estimates. In the MI analyses, either with or without the genetic variant, we used 5 imputations taken from evenly spread points with the Monte Carlo chain and each new dataset was regressed on $\mathbf{S}$ and $\mathbf{X}$ using a LMM.

For each set of simulations, we calculated: the bias in the coefficient of the genetic effect (bias), i.e. the difference between the average value of the coefficient and the true value; the root mean square error (rmse), i.e. the square root of the average of the square of the difference between the coefficient and the true value; the coverage of the genetic effect estimate (cp), i.e. the proportion of time that the true genetic effect falls within the $95 \%$ confidence interval (credible interval) of the estimate; Lin's concordance correlation coefficient (Lc), which measures the agreement between the estimate obtained from the uncensored dataset and the estimate obtained from the dataset after the censoring [Lin, 1989].

Among all modelling solutions, the Tobit with robust standard errors was the only one that did not model the family structure explicitly. To assess its robustness to different trios composition, we performed a sensitivity analysis considering trios composed of two parents and one child so that the matrix $\mathbf{K}_{i}=\left(\begin{array}{ccc}1 & 0 & 0.5 \\ 0 & 1 & 0.5 \\ 0.5 & 0.5 & 1\end{array}\right)$. We simulated 1,000 datasets as before but with $\rho_{X S}=0$ and $\rho_{X Y}=0.5$. In each dataset, $40 \%$ of observations were left-censored.

All the analyses were performed using the software R version 2.10.0 (http://www . R-project .org/).

Table 3 shows the results for the scenario illustrated in Figure 2a. As expected, the simple substitution approach, where ND values were replaced by the LOD, gave biased results with small standard errors and a low coverage probability, especially for a censoring rate of $40 \%$. These results were accompanied by low concordance between the estimate from the uncensored data and those obtained after censoring, with a Lin's coefficient of $90 \%$ and $63 \%$ for a censoring rates of $20 \%$ and $40 \%$, respectively. Results were similar when ND values were replaced by LOD/2 (data not shown). Our simulations suggested that simple substitution tends to bias the estimate of the genetic effect towards the null. The bias was proportional to the censoring rate, but it did not change with the correlation between $\mathbf{X}$ and $\mathbf{Y}$. 
The Tobit method with robust standard error gave less biased estimates for any level of censoring, with larger rmse. The coverage probability was about 94\%. The Lin's coefficient was not smaller than $96 \%$ when the censoring rate was $20 \%$, and not smaller than $91 \%$ when censoring rate was $40 \%$. The Tobit performed similarly well in the sensitivity analysis based on families of two parents and one child instead of three siblings: with a censoring rate of $40 \%$ and a correlation between $\mathbf{X}$ and $\mathbf{Y}$ equal to 0.5 , the bias of the estimate was 0.001 with coverage rate of $95 \%$ and Lin's coefficient of $93 \%$.

The Bayesian analyses gave slightly biased estimates with smaller rmse. These results are supported by coverage probabilities equal to $95 \%$. The Lin's coefficient was not smaller than $97 \%$ with $20 \%$ of censoring, and not smaller than $90 \%$ with $40 \%$ of censoring.

The MI approaches provided stable results using 5 imputations. The average estimates were slightly biased and similar to the Bayesian ones, with smaller coverage probabilities and Lin's coefficients. Moreover, they performed better with a censoring rate of $20 \%$. The exclusion of the genetic variant from the imputation model did not increase the bias.

Results from all strategies slightly changed when increasing the correlation between $\mathbf{X}$ and Y. As expected, they became worse with a $40 \%$ censoring rate compared to a $20 \%$ censoring rate.

The results of the second scenario, where we introduced a positive correlation between $\mathbf{X}$ and $\mathbf{S}$, are reported in Table 4 . Results did not change by varying the correlation.

Table 3: Association between the censored biomarker $\mathbf{Y}$ and the genetic variant $\mathbf{S}$ in presence of family structure, when $\mathbf{S}$ is not correlated to other covariates of $\mathbf{Y}$ (Figure 2a). Results are reported by level of correlation between $\mathbf{X}$ and $\mathbf{Y}$, and for two degrees of censoring, $20 \%$ and $40 \%$.

\begin{tabular}{|c|c|c|c|c|c|c|c|c|c|c|c|c|c|}
\hline \multirow[b]{3}{*}{ Method } & \multirow[b]{3}{*}{ Censoring \% } & \multicolumn{12}{|c|}{ Correlation between $\mathbf{X}$ and $\mathbf{Y}$} \\
\hline & & \multicolumn{4}{|c|}{0.2} & \multicolumn{4}{|c|}{0.5} & \multicolumn{4}{|c|}{0.8} \\
\hline & & bias & rmse & $c p$ & $L c$ & bias & rmse & $c p$ & $L c$ & bias & rmse & $c p$ & $L c$ \\
\hline Complete & 0 & -0.001 & 0.085 & 95 & & -0.005 & 0.089 & 94 & & -0.005 & 0.086 & 95 & \\
\hline \multirow[t]{2}{*}{ 1. Substitution } & 20 & -0.030 & 0.078 & 92 & 89 & -0.033 & 0.083 & 91 & 90 & -0.035 & 0.081 & 92 & 89 \\
\hline & 40 & -0.061 & 0.084 & 81 & 63 & -0.059 & 0.084 & 82 & 64 & -0.061 & 0.087 & 81 & 64 \\
\hline \multirow[t]{2}{*}{ 2. Tobit } & 20 & -0.001 & 0.087 & 94 & 97 & -0.005 & 0.091 & 93 & 97 & -0.008 & 0.088 & 95 & 96 \\
\hline & 40 & -0.002 & 0.090 & 95 & 93 & 0.001 & 0.096 & 94 & 93 & -0.004 & 0.096 & 94 & 91 \\
\hline \multirow[t]{2}{*}{ 3. Bayesian } & 20 & -0.004 & 0.086 & 95 & 97 & -0.009 & 0.090 & 94 & 98 & -0.014 & 0.086 & 94 & 97 \\
\hline & 40 & -0.012 & 0.086 & 94 & 93 & -0.010 & 0.091 & 95 & 93 & -0.020 & 0.089 & 95 & 90 \\
\hline \multirow[t]{2}{*}{ 4. $\mathrm{MI}+\mathrm{S}$} & 20 & -0.005 & 0.087 & 92 & 97 & -0.009 & 0.090 & 91 & 97 & -0.014 & 0.087 & 92 & 96 \\
\hline & 40 & -0.013 & 0.088 & 90 & 90 & -0.010 & 0.094 & 90 & 90 & -0.020 & 0.092 & 89 & 88 \\
\hline \multirow[t]{2}{*}{ 5. MInoS } & 20 & -0.003 & 0.087 & 93 & 96 & -0.007 & 0.092 & 91 & 97 & -0.012 & 0.088 & 91 & 96 \\
\hline & 40 & -0.011 & 0.088 & 92 & 90 & -0.011 & 0.093 & 91 & 88 & -0.021 & 0.092 & 91 & 87 \\
\hline
\end{tabular}

The table reports: bias of the genetic effect estimates (bias), root mean square error (rmse), coverage probability (cp) of the 95\% confidence interval of the estimate, and Lin's concordance correlation coefficient between the estimate obtained with the complete dataset (ideal value) and the estimate obtained after censoring (Lc). Coverage probability and Lin's coefficient values are in percentage. The value assumed for the genetic effect is 0.15 . 
Table 4: Association between the censored biomarker $\mathbf{Y}$ and the genetic variant $\mathbf{S}$ in presence of family structure, when $\mathbf{S}$ is correlated to other covariates of $\mathbf{Y}$ (Figure $2 \mathrm{~b}$ ). Results are reported for a fixed level of correlation between $\mathbf{X}$ and $\mathbf{Y}\left(\rho_{X Y}=0.2\right)$, by levels of correlation between $\mathbf{S}$ and $\mathbf{X}$, and for two degrees of censoring, $20 \%$ and $40 \%$.

\begin{tabular}{|c|c|c|c|c|c|c|c|c|c|}
\hline \multirow[b]{3}{*}{ Method } & \multirow[b]{3}{*}{ Censoring \% } & \multicolumn{8}{|c|}{ Correlation between $\mathbf{S}$ and $\mathbf{X}$} \\
\hline & & \multicolumn{4}{|c|}{0.3} & \multicolumn{4}{|c|}{0.5} \\
\hline & & bias & rmse & $c p$ & $L c$ & bias & rmse & $c p$ & $L c$ \\
\hline Complete & 0 & -0.003 & 0.084 & 95 & & -0.002 & 0.086 & 96 & \\
\hline \multirow[t]{2}{*}{ 1. Substitution } & 20 & -0.031 & 0.077 & 93 & 89 & -0.030 & 0.079 & 93 & 89 \\
\hline & 40 & -0.060 & 0.085 & 81 & 65 & -0.057 & 0.082 & 83 & 63 \\
\hline \multirow[t]{2}{*}{ 2. Tobit } & 20 & -0.003 & 0.086 & 96 & 96 & -0.001 & 0.087 & 95 & 97 \\
\hline & 40 & -0.003 & 0.095 & 94 & 93 & 0.002 & 0.093 & 94 & 92 \\
\hline \multirow[t]{2}{*}{ 3. Bayesian } & 20 & -0.006 & 0.085 & 96 & 97 & -0.005 & 0.086 & 95 & 98 \\
\hline & 40 & -0.012 & 0.090 & 95 & 93 & -0.008 & 0.088 & 95 & 93 \\
\hline \multirow[t]{2}{*}{ 4. $\mathrm{MI}+\mathrm{S}$} & 20 & -0.006 & 0.086 & 92 & 96 & -0.005 & 0.088 & 92 & 97 \\
\hline & 40 & -0.013 & 0.092 & 91 & 91 & -0.008 & 0.090 & 92 & 90 \\
\hline \multirow[t]{2}{*}{ 5. MInoS } & 20 & -0.004 & 0.088 & 93 & 96 & -0.003 & 0.089 & 93 & 96 \\
\hline & 40 & -0.013 & 0.093 & 90 & 90 & -0.008 & 0.089 & 93 & 90 \\
\hline
\end{tabular}

The table reports: bias of the genetic effect estimates (bias), root mean square error (rmse), coverage probability (cp) of the 95\% confidence interval fo the estimate, and Lin's concordance correlation coefficient between the estimate obtained with the complete dataset, i.e. the ideal value, and the estimate obtained after censoring (Lc). Coverage probability and Lin's coefficient values are in percentage. The value assumed for the genetic effect is 0.15 .

\section{Discussion}

Despite the growing attention to the problem of censoring and the fact that biomarkers are quite often subject to censoring as a consequence of detection limits in the laboratory assays, censored data methods have received limited attention in genetic association studies and they are not routinely applied. This paper illustrates different approaches to deal with censoring in the context of genetic association studies by using real and simulated data.

We provide a real data application analysing serum transferrin levels in a subsample of 393 individuals from the MICROS study, considering three genetic variants with different allele frequency, modeled one by one, as usually processed in genetic association studies. The results suggest that the best strategy for dealing with censored biomarkers is the Bayesian analysis, which is the more stable method providing estimates very close to those obtained with the complete dataset, for any level of censoring. The Tobit method with robust standard errors in the presence of family structure could lead to overestimation of the genetic effect and correspondingly to smaller p-values in case of high censoring rates. Thus, in a GWAS context, using Tobit with high censored levels might produce an increased number of false discoveries. Both MI approaches, including or excluding the genetic variant, could produce good estimates. The simple substitution seems to provide the most biased results, except when the allele frequency of genetic variant is large. However, additional simulations should be performed to investigate the impact on the results of varying the allele frequency and of a joint analysis using the genetic variants all together.

Through simulations we found that estimates of the genetic effect obtained by simply 
deleting censored data are biased towards the null hypothesis, with low coverage probabilities, which are reduced to $56 \%$ and $34 \%$ for censoring rate of $20 \%$ and $40 \%$, respectively (data not shown). However, findings confirm that if the censoring rate is lower than $10 \%$, all strategies including deletion of ND values, lead to similar results (data not shown) [Lubin et al., 2004].

Our simulations also suggest that the Tobit model with robust standard errors could be a good approach to deal with censoring when the data are dependent because of the presence of a family structure. Indeed, the strategy gives unbiased results when the censoring rate is equal to $20 \%$, and even at a higher censoring rate the bias is small. It should be investigate further how the Tobit with robust standard errors performs in the presence of more complex family structure.

In this paper we adopt a Bayesian analysis, which allows us to take into account the family structure of the sample. For all levels of censoring such an approach gives slightly biased results, with correct standard errors. This slightly negative bias could be due to the influence of the zero centred priors. The Bayesian framework is flexible and could be extended to more complex family structures. Our simulations show that the Bayesian analysis represents a good strategy for candidate gene association analysis.

We also propose a MI approach, where the Bayesian model structure is used only for the imputation phase of censored data, followed by LMM estimation. The results are close to those obtained with the Bayesian model.

The MI approach could be applied either in the context of a candidate gene analyses or in a GWAS, where millions of genetic variants are investigated. Including covariates in the MI model is recommended because it improves parameter estimation in most cases [White et al., 2011]. The imputation model should include all variables of the analysis: those highly correlated with response variable and those that explain the mechanism leading to censored data [Collins et al., 2001, Bell and Fairclough, 2013]. But, in a GWAS context, the application of a correctly specified MI procedure is going to be computationally infeasible. In fact, each single genetic variant should be included in the imputation process and each imputation should be repeated at least three times to guarantee that the uncertainty of the imputation process is taken into account. For these reasons, we explored the performance of MI when the genetic variant is not included in the imputation model. Through simulation we found that the MI approach without the genetic variant gives estimates similar to those obtained with the MI including the genetic variant and the full Bayesian approach. Beyond the computational considerations, the choice of not including the genetic variant in the MI model is supported by the evidence that individual variants explain a negligible proportion of the variance of complex disease biomarkers [Manolio et al., 2009]. Such a choice might be reconsidered in the case of biomarkers that are controlled by a major gene and thus a specific variant may excerpt a large effect. However, in such a situation, it could be recommended to include the major gene variant into the MI model, but not each single genetic variant. In fact, no other variant is expected to have a large effect on the biomarker after the major gene variant has been taken into account.

While the results from the simulation show good average performance of the Bayesian and MI-based approaches, in the empirical example the performance varies according to the 
genetic variant considered. This is related to the small sample size and the fact that we are no longer looking at average performance.

On the basis of our simulations and real data analysis, we conclude that, in the context of a genome-wide analysis, approaches that incorporate the genetic variant into the likelihood estimation, such as the full Bayesian model or the MI procedure including the genetic variant, are not practically feasible due to their high computational demand. We recommend either the Tobit approach especially when the censoring rate is moderate given its computational simplicity, or the MI without the genetic variant. Whereas, in the context of candidate gene analysis, we suggest the Bayesian analysis for any level of censoring.

Our study has some limitations that warrant further work. A limitation is the constant family size, which we chose to simplify the model framework in order to compare a wider range of scenarios. Exploration of more complex family structures should be considered to assess the impact of different types of dependency on effect estimates and standard errors.

Non-informative priors were used in both the simulation work and the real data example. These are often used as a 'reference priors'. Clearly, it would be possible to include informative priors provided that they took the conjugate form. Strong prior knowledge would affect the solution, but it would also make it impossible to compare the Bayesian results with those of the other approaches that cannot use prior information. In such familial context, it might also be valuable to explore different trade-offs between genetic and non-genetic variance for the Bayesian model formulation. In our application, we chose to set the genetic variance equal to the estimated genetic heritability of serum transferrin in the whole MICROS study sample. Given the large sample size, such an estimate should be considered as a very reliable value. However, two caveats should be mentioned. The first one is that, even if 0.35 is a good approximation for the heritability of many routinely measured blood markers [Marroni et al., 2008], different biomarkers are characterized by different genetic heritabilites and so a broader spectrum of possible heritability values should be analyzed to make our results more generalizable. A second caveat is that we intrinsically assumed the genetic variance to be completely explained by the polygenic additive effect of multiple loci. This assumption does not take into account the effect of non-additive genetic components such as, for instance, gene-gene interactions. However, non-additive genetic effects are rarely taken into account in genetic heritability analysis because they are difficult to model in the absence of specific knowledge related to clearly identified gene mechanisms.

Finally, the focus of the paper is on left-censored data, but the study framework could be extended to right or interval censoring. The assumption of normality of the response variable distribution can often be satisfied with appropriate transformations. However, in the presence of non-normal data a Bayesian non-parametric approach could also be explored, or alternatively, a Bayesian analysis could be extended to encompass mixtures of normal distributions.

Collaboration between EURAC and the University of Leicester was supported by a travel grant from the Royal Society (International Exchanges Scheme). The authors are grateful to Dr. Prof. Peter P. Pramstaller for the data of the MICROS study, Martin Gögele for the data extraction, Yuri D'Elia for his computer and technical support, and Federico Murgia 
for the heritability estimation.

\section{Conflict of Interest}

The authors have declared no conflict of interest.

[Bell and Fairclough, 2013] Bell, M. L. and Fairclough D.L. (2013). Practical and statistical issues in missing data for longitudinal patient reported outcomes. Stat Methods Med Res Feb 19.

[Box and Tiao, 2011] Box, G. E. P. and Tiao G. C. (2011). Bayesian inference in statistical analysis. John Wiley and Sons.

[Casella and Berger, 2002] Casella, G. and Berger, R. L. (2002). Statistical Inference. Second Edition. R.L., Thomson Learning.

[Collins et al., 2001] Collins, L. M., Schafer, J. L. and Kam, C. (2001). A comparison of inclusive and restrictive strategies in modern missing data procedures. Psychological Methods 6, 330-351.

[Del Greco-M et al., 2011] Del Greco-M. F., Pattaro, C., Luchner, A., Pichler, I., Winkler, T., et al. (2011). Genome-wide association analysis and fine mapping of NT-proBNP level provide novel insight into the role of the MTHFR-CLCN6-NPPA-NPPB gene cluster. Hum Mol Genet 20, 1660-1671.

[Diao et al., 2004] Diao, G., Lin, D. Y. and Zou, F. (2004). Mapping quantitative trait loci with censored observations. Genetics 168, 1689-1698.

[Diao and Lin, 2006] Diao, G. and Lin, D. Y. (2006). Semiparametric variance-component models for linkage and association analyses of censored trait data. Genetic Epidemiology 30, 570-581.

[Dipak et al., 2000] Dipak, K. D., Sujit K. G., and Bani K. M. (2000). Generalized Linear Models: A Bayesian Perspective, Chapman and Hall/CRC Biostatistics Series.

[El Ghouch and Van Keilegom, 2009] El Ghouch, A. and Van Keilegom, I. (2009). Local linear quantile regression with dependent censored data. Statistica Sinica 19, 1621-1640.

[Epstein et al., 2003] Epstein, M. P., Lin, X., and Boehnke M. (2003). A Tobit variance-component method for Linkage Analysis of censored trait data. Am J Hum Genet 72, 611-620.

[Fang, 2006] Fang, Y. (2006). A note on QTL detecting for censored traits. Genet Sel Evol 38, 221-229.

[Flury, 1997] Flury, B. (1997). A First Course in Multivariate Statistics. Springer.

[Gelfand et al., 1990] Gelfand, A. E., Hills, S. E., Racine-Poon A. and Smith, A. F. M. (1990). Illustration of Bayesian inference in normal data models using Gibbs sampling. Journal of the American Statistical Association 85, 972-985.

[Gelman et al., 2004] Gelman, A., Carlin, J. B., Stern, H. S. and Rubin, D. B. (2004). Bayesian Data Analysis. Second edition, Chapman and Hall.

[Gögele et al., 2011] Gögele, M., Pattaro, C., Fuchsberger, C., Minelli, C., Pramstaller, P.P., and Wjst, M. (2011). Heritability analysis of life span in a semi-isolated population followed across four centuries reveals the presence of pleiotropy between life span and reproduction. J Gerontol A Biol Sci Med Sci 66, 26-37.

[Heitjan, 1993] Heitjan, D. (1993). Ignorability and coarse data: some biomedical examples. Biometrics 49, 1099-1109.

[Heitjan and Rubin, 1991] Heitjan, D. and Rubin, D. B. (1991). Ignorability and coarse data. Annals of Statistics 19, 2244-2253.

[Hsu, 2007] Hsu, C. H., Taylor, J. M. G., Murray, S. and Commenges, D. (2007). Multiple imputation for interval censored data with auxiliary variables. Stat in Med 26, 769-781.

[Li et al., 2006] Li, M., Boehnke, M., Abecasis, G. R. and Song, P. X. (2006). Quantitative trait linkage analysis using Gaussian copulas. Genetics 173, 2317-2327.

[Li et al., 2010] Li, M., Reilly, C. and Hanson, T. (2010). Association Tests for a Censored Quantitative Trait and Candidate Genes in Structured Populations with Multilevel Genetic Relatedness. Biometrics 66, 925-933.

[Lin, 1989] Lin, L. I-K. (1989). A concordance correlation coefficient to evaluate reproducibility. Biometrics 45, 255-268.

[Little and Rubin, 2002] Little, R. J. A. and Rubin, D. B. (2002). Statistical Analysis with Missing Data. Second edition, New York: John Wiley and Sons. 
[Lubin et al., 2004] Lubin, J. H., Colt, J. C., Camann, D., Davis, S., Cerhan, R. J., et al. (2004). Epidemiologic evaluation of measurement data in the presence of detection limits. Environ. Health Perspectives 112, 1691-1696.

[Marroni et al., 2008] Marroni, F., Grazio, D., Pattaro, C., Devoto, M., Pramstaller, P. (2012). Estimates of genetic and environmental contribution to 43 quantitative traits support sharing of a homogeneous environment in an isolated population from South Tyrol, Italy. Hum Hered 65, 175-182.

[Manolio et al., 2009] Manolio, T. A., Collins, F. S., Cox, N.J., Goldstein, D.B., Hindorff, L.A., et al. (2009). Finding the missing heritability of complex diseases. Nature 461, 747-753.

[Neal, 2003] Neal, R. M. (2003). Slice sampling. The Annals of Statistics 31, 705-767.

[Pattaro et al., 2007] Pattaro, C., Marroni, F., Riegler, A., Mascalzoni, D., Pichler, I., et al. (2007). The genetic study of three population microisolates in South Tyrol (MICROS): study design and epidemiological perspectives. BMC Med Genet 5, 8-29.

[Pichler et al., 2011] Pichler, I., Minelli, C., Sanna, S., Tanaka, T., Schwienbacher, C., et al. (2011). Identification of a common variant in TFR2 gene implicated in the physiological regulation of serum iron levels. Hum Mol Genet 26, 1232-1240.

[Portonoy, 2003] Portonoy, S. (2003). Censored quantile regression. The Journal of the American Statistical Association 98, 1001-1012.

[Price et al., 2006] Price, A. L., Patterson, N. J., Plenge, R. M., Weinblatt, M. E., Shadick, N. A. and Reich, D. (2006). Principal components analysis corrects for stratification in genome-wide association studies. Nat Genet 38, 904-909.

[Rubin, 1996] Rubin, D. B. (1996). Multiple imputation after 18+ years. Journal of American Statistical Association 91, 473-489.

[Schafer, 1997] Schafer, J. L. (1997). Analysis of Incomplete Multivariate Data. New York: John Wiley and sons.

[Schafer, 1999] Schafer, J. L. (1999). Multiple imputation: A primer. Stat Methods Med Res 8, 3-15.

[Schafer et al., 2002] Schafer, J. L. and Graham, J. W. (2002). Missing data: our view of the state of art. Psychol Methods 7, 147-177.

[Schafer and Yucel, 2002] Schafer, J. L. and Yucel R. M. (2002). Computational strategies for multivariate linear mixed-effect models with missing values. Journal of Computational and Graphical Statistics 11, 437-457.

[Schifano et al., 2012] Schifano, E. D., Epstein, M. P., Bielak, L. F., Jhun, M. A., Kardia, S. L. R., et al. (2012). SNP Set Association Analysis for Familial Data. Genetic Epidemiology 36, 797-810.

[Tanner, 1991] Tanner, M. A. (1991). Tools for statistical inference (Vol. 3). New York: Springer.

[Taylor, 1987] Taylor, J. K. (1987). Quality Assurance of Chemical Measurements, Chelsea, MI: Lewis Publishers.

[Tobin, 1958] Tobin, J. (1958). Estimation of relationships for limited dependent variables. Econometrica 26, 24-36.

[Visscher et al., 2004] Visscher, P. M., Benyamin B. and White I. (2004). The use of linear mixed models to estimate variance components from data on twin pairs by maximum likelihood. Twin Research $\mathbf{7}$, 670-674.

[White and Carlin, 2010] White, I. R. and Carlin, J. B. (2010). Bias and efficiency of multiple imputation compared with complete-case analysis for missing covariate values. Stat in Med 29, 2920-2931.

[White et al., 2011] White, I. R., Royston, P. and Wood, A. M. (2011). Multiple imputation using chained equations: Issues and guidance for practice. Stat in Med 30, 377-399.

[Wooldridge, 2002] Wooldridge, J. M. (2002). Econometric Analysis of Cross Section and Panel Data. MIT Press, Cambridge.

[Yucel, 2008] Yucel, R.M. (2008). Multiple imputation inference from multivariate multilevel continuous data with ignorable nonresponse. Phil. Trans. R. Soc. A, 366, doi : 10.1098/rsta.2008.0038. 